\title{
Analysis on Fracture Reason of 20CrMo Driving Shaft
}

\author{
Jiao Yunjing ${ }^{1,2}$ \\ ${ }^{1}$ North China Institute of Aerospace Engineering \\ Langfang, 'China \\ ${ }^{2}$ Mechanical institute, Tianjin university, \\ Tianjin, China \\ Email:Jiaoyj1234567@163.com
}

\author{
Sun Mei \\ Tianjin Testing and Calibration Center \\ Tianjin, China \\ Flying_123@163.com
}

\begin{abstract}
The fracture reason of a driving shaft used motorcycle engine was analyzed by means of macroscopic examination, chemical analysis, fracture analysis, hardness testing. The results showed that the fatigue fracture mode was determined and incomplete hardening of the axis and increasingly growing load were the main reasons which accelerated the fatigue fracture of the driving shaft.
\end{abstract}

Keywords-fracture; martensite; carburizing; quenching; driving shaft

\section{INTRODUCTION}

A motorcycle engine driving shaft whose material is $20 \mathrm{CrMo}$. The driving axle matched with original 10-inch wheels; now we need to use the driving axle to match the new engine with12-inch wheels. Compared with the original engine, the engine power increased $8 \%$ than the original engine power. At first, the calculation was conducted on the static strength, fatigue strength and its stability. The result of calculation showed that the driving shaft was safe to match the new machine. But in actual operation, during the 600 hours motorcycle chassis durability test, the driving shaft fracture occurred twice. In this paper, the fracture reason was analyzed from the material aspects in the case of existing equipment.

While failure analysis was carried on from the material aspects, because the chemical composition and microstructure decided the using performance, so failure analysis should be carried in the order : ingredient $\rightarrow$ Organization $\rightarrow$ performance[1-7].

\section{CHEMICAL ANALYSIS}

\section{A. Macroscopic fracture analysis}

The macro-morphology of the broken pieces is shown in Figure 1. From the fig. 1 we can know there is obvious plastic deformation in the fracture and the fracture surface include fatigue source region, fatigue crack growth area and instantaneous fracture zone[7]. So, we judged that the fracture was fatigue fracture.

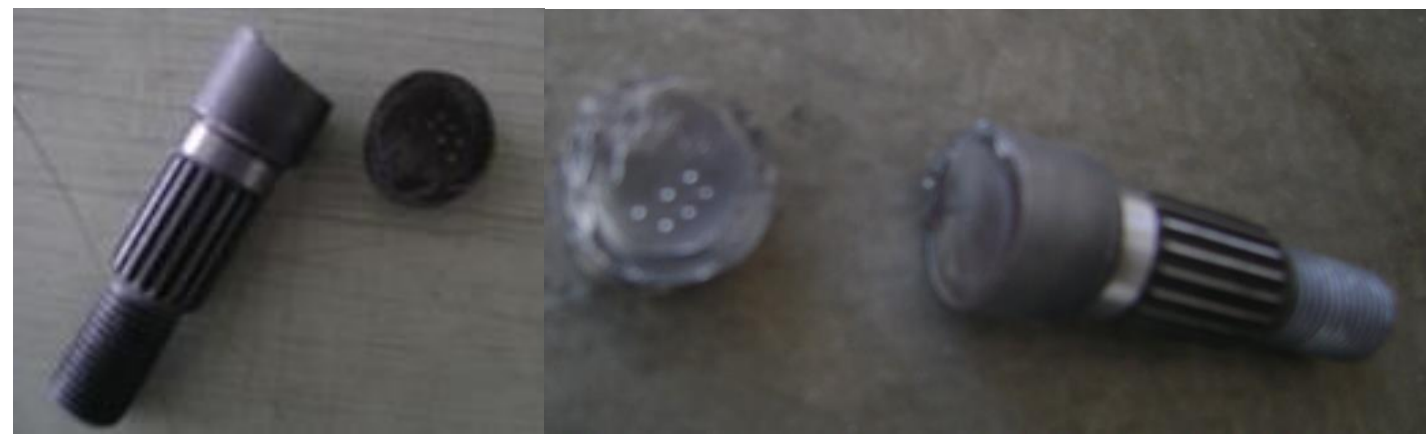

Figure 1 Appearance of fractured driving shaft

\section{B. Analysis of the chemical composition}

Spectroscopic chemical analysis on sampling near the fracture driving shaft was carried on as shown in Figure2. The spectrometer is model of OBLF-750 and made in Germany. The result of analysis is shown in Table 1. Compared with the standard material composition, the chemical composition of the driving shaft is in line with the provisions of GB3077-88 of 20CrMo steel. So the chemical elements of raw materials are qualified. 


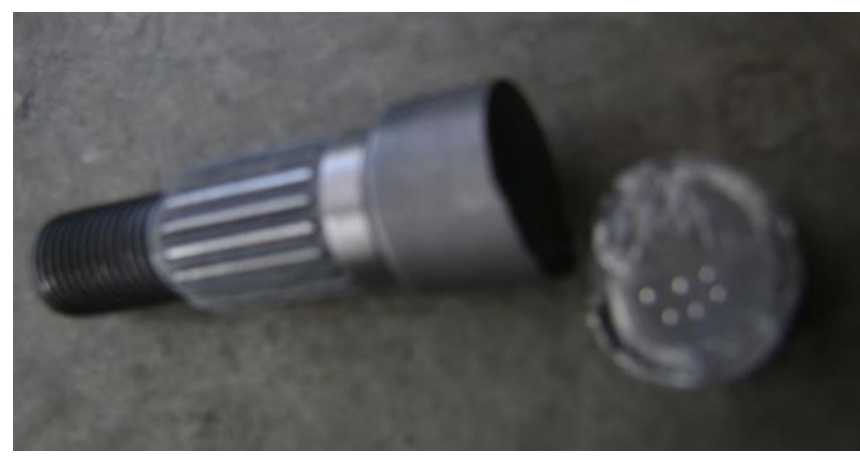

Figure 2 Spectroscopic chemical analysis

TABLE.I CHEMICAL COMPOSITION OF THE CORE OF FRACTURED DRIVING SHAFT（ \% )

\begin{tabular}{lccccccc}
\hline Composition & $\mathrm{C}$ & $\mathrm{Si}$ & $\mathrm{Mn}$ & $\mathrm{P}$ & $\mathrm{S}$ & $\mathrm{Cr}$ & $\mathrm{Mo}$ \\
\hline Driving shaft & 0.22 & 0.26 & 0.50 & 0.012 & 0.016 & 0.86 & 0.17 \\
\hline GB3077-88 & $0.17 \sim 0.2$ & $0.17 \sim 0.37$ & $0.40 \sim 0.70$ & $\leqslant$ & $\leqslant$ & $0.80 \sim 1.10$ & $0.15 \sim 0.25$ \\
& 4 & & & 0.035 & 0.035 & & \\
\hline
\end{tabular}

\section{Metallographic examination}

The heat-treatment of the driving shaft includes carbonitriding, quenching and tempering. The sample was gotten near the fracture source region. The cross section of the sample was used to prepare the observed surface and the inlaid sample was milled in the 200-1200 number of sandpaper. After finished sample grinding, the sample was rough polished fine polished until the sample was looked bright as a mirror without any minor scratches, as shown in fig.3. Using alcohol with $4 \%$ nitric acid solution eroded the surface of the sample, then the erosion good sample was observed using Carl Zeiss inverted microscope which model was Axiovert 40 MAT. The sample of fracture shaft was observed microstructure of the surface layer and the core section, as shown in Figure4 and Fig .5. The surface structure of specimen was shown in Figure4 and core tissue sample was shown in Figure5. From the fig. 4 we can learn that the surface microstructure was tempered martensite (tempering $\mathrm{M}$ ) and a small amount of residual austenite (A ') and the structure was normal; from the fig. 5 we can learn that the core part was ferrite microstructure $(\mathrm{F})$ and pearlite $(\mathrm{P})$, and the structure of core is the balance organization[8-10].

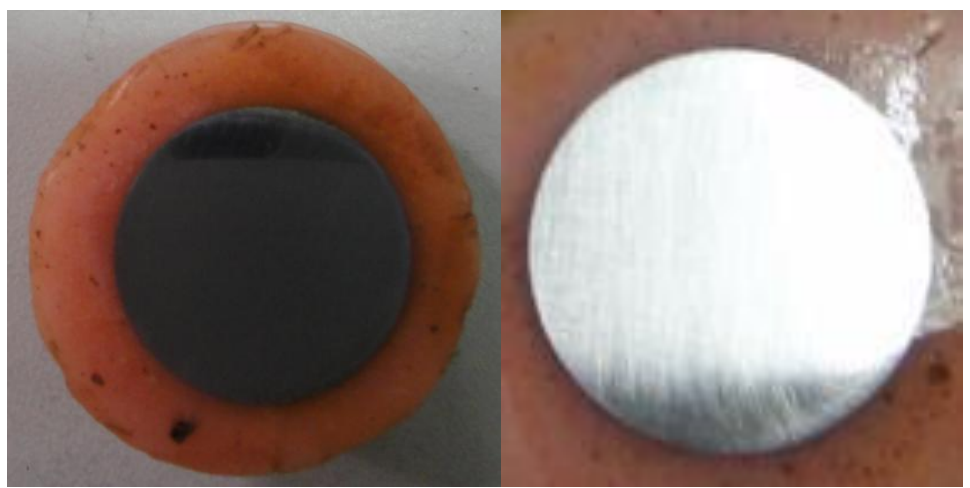

Figure 3 Sample of Metallographic examination 


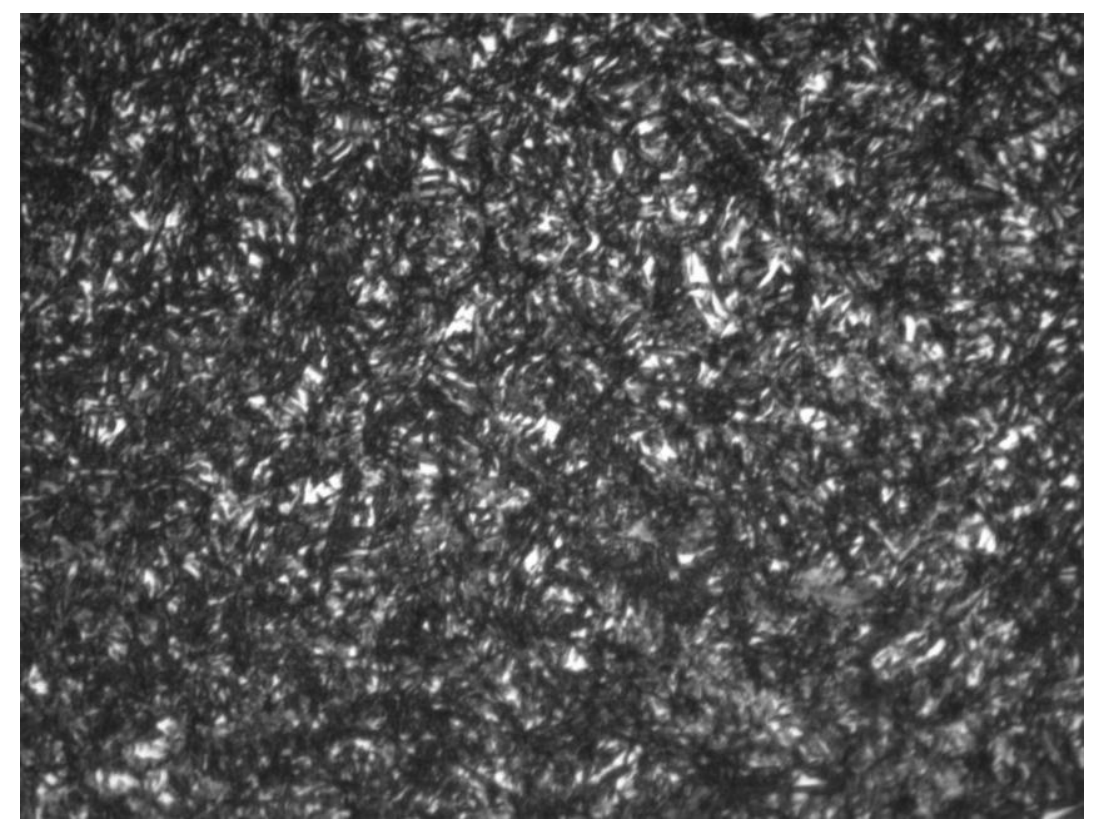

Figure 4 Surface texture of fracture driving shaft: tempering $\mathrm{M}+\mathrm{A}^{\prime} \sim 540 \mathrm{X}$

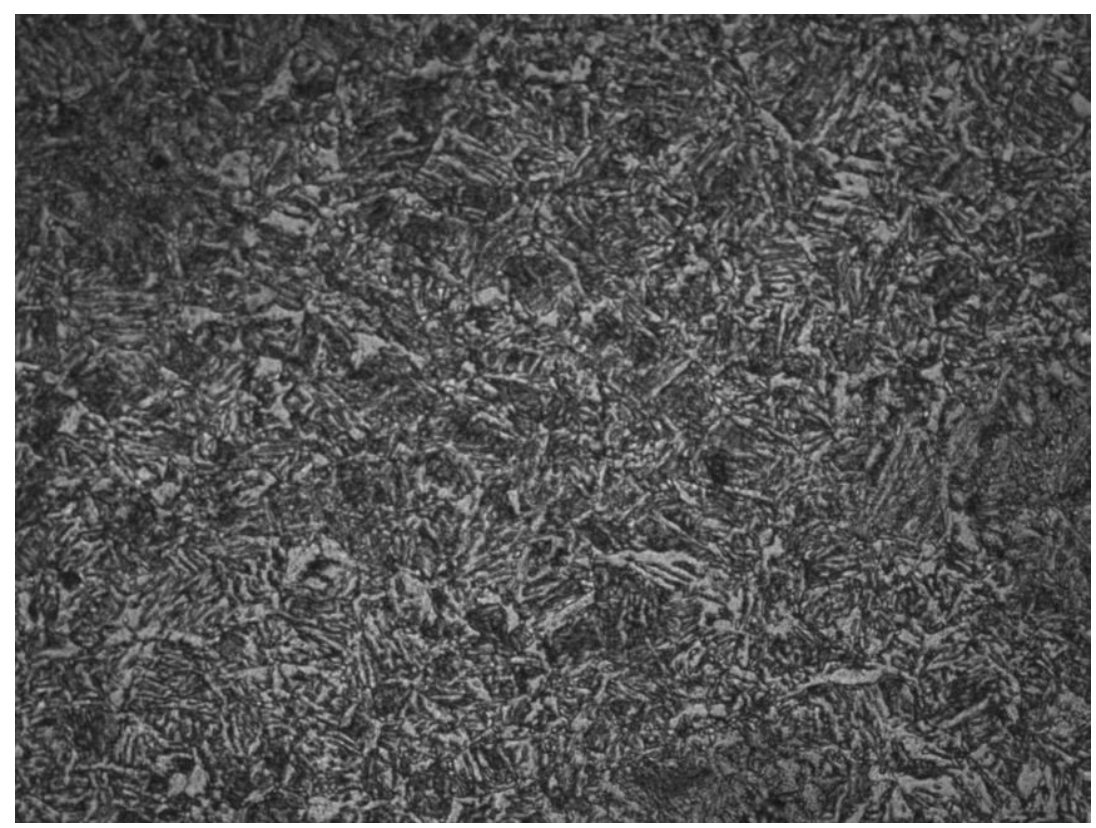

Figure 5 Core texture of fracture driving shaft: $\mathrm{F}+\mathrm{P} \sim 540 \mathrm{X}$

\section{Hardness Testing}

Using the HVRV-187.5-type Brinell hardness gauge, the surface and the core part was measured as shown in fig.6; and the demand of measurement includes two hands: Load of $1471 \mathrm{~N}$ and hold time for 8-10s. The result of measurement is shown in the Table2.

As we can learn from the Table 2, the surface hardness values meet the design requirements and the core hardness values are significantly lower than the design requirements.

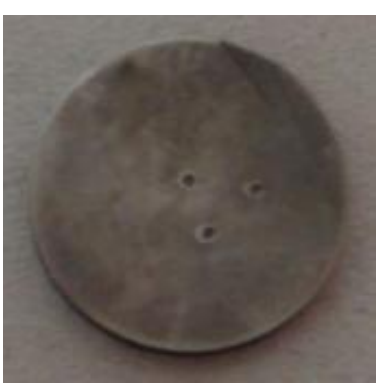

Figure 6 Sample of hardness 
TABLE II SURFACE AND CORE HARDNESS OF FRACTURED DRIVING SHAFT

\begin{tabular}{cccc}
\hline part of hardness test & Measure value & Mean value & Desired value \\
\hline External round surface(HRA) & $81,83.5 、 80.5$ & 81.67 & $78 \sim 83$ \\
\hline Core of cross section (HRC) & $20.5 、 20 、 21$ & 20.5 & $28 \sim 43$ \\
\hline
\end{tabular}

\section{ANALYSIS AND DISCUSSION}

Based on the above physical and chemical test results, the chemical composition of the driving shaft is of qualified. Viewed from the microstructure, hardness of sample core matched with the microstructure match. The heat-treatment process includes carburizing quenching and tempering. We can know from the design drawings that the heat-treatment process of the fracture shaft was carried on carbonitriding, quenching and tempering. But the hardness of core part is lower than the demanded value. In cording to the demanded hardness value, the core texture should include higher hardness tempered martensite and tempered troostite texture.

\section{CONCLUSION}

Through analysis and testing, the results showed that the hardenability of driving shaft was not qualified and the strength in core part was low. In a word, the fatigue reason of the driving shaft is because of the bad hardenability and the growing load.

\section{ACKNOWLEDGEMENT}

Langfang City Science and Technology Support Program, Project number: 2014011054

\section{REFERENCES}

[1] Chen $\mathrm{Yu}$, Failure Analysis on Fracture of Motorcycle Spindles[J].Physical testing and chemical analysis partA:Physical testing.2012,48 (4):272-278.

[2]Wu Junfeng, Reason Analysis on Cracking of Unilateralism Organ Axes of 20Cr Steel, Physical testing and chemical analysis partA:Physical testing.2012,48 (1):59-61

[3]Wan Youjuan, Luo Yuansheng, Yang Xiaomin, Fracture Analysis on Welded Joints of Rotatable Bracket, Physical testing and chemical analysis partA:Physical testing.2012,48 (1):55-58

[4]Yang Chuanzheng,Reasonable Using Various Kinds of Test Research Methods in Material Analysis, Physical testing and chemical analysis partA:Physical testing.2012,48 (4):279-282

[5]Shi Qiang, Wang Weiqiang, Tang Jie,Kong Haoyuan, Analysis on Fracture Reason of Bolt on Poston Compressor Coupling, Physical testing and chemical analysis partA:Physical testing.2012,48 (3):203-205

[6]Yu Qizhong,Fracture Analysis on Binding Bolt of Crane Turnplate, Physical testing and chemical analysis partA:Physical testing.2012,48 (3):194-196

[7] Zuo Huafu,Jiang Yuanyuan ,Liu Xiaohong, Fatigue Failure Analysis on End Reduction Input Gearwheel[J].Physical testing and chemical analysis partA:Physical testing.2012,48 (2):120-124

[8]Ministry of Aerospace Industry Aerospace Equipment Failure Analysis Center, metallic material fracture analysis and mapping [M] Beijing: Science Press.

[9]Shi Meitang, Metal Material And Heat Treatment.[M].Shanghai: Shanghai Science and Technology Press, 1984.

[10]Guo Daren, Fracture and Fracture Analysis of Metal Materials, Foreign metal heat treatment,1996,4. 